\title{
Spinocerebellar Ataxia Type 6 (SCA6) Phenotype in a Patient with an Intermediate Mutation Range CACNA1A Allele
}

\section{Leslie Manace Brenman*}

University of California, Sanfrancisco, California, USA

\begin{abstract}
Spinocerebellar ataxia type 6 (SCA6) is one of multiple autosomal dominant progressive ataxias due to an expanded trinucleotide repeat gene sequence. Characterization of normal and pathogenic trinucleotide (or triplet) repeat sizes in the gene associated with SCA6, CACNA1A, is ongoing. Normal alleles contain 18 or fewer CAG repeats and full-penetrance alleles contain 20 to 33 triplet repeats; 19 CAG repeats seem to have variable effect. Meiotic expansion from the 19-CAG repeat allele to full pathogenic range has been reported. Affected individuals homozygous for this intermediate 19-CAG repeat allele and asymptomatic individuals up to late age-heterozygous for this intermediate allele have also been reported. We present a case report of a 44-year-old woman heterozygous for 19 CAG repeats with normal remaining gene sequence in CACNA1A presenting with an SCA6 phenotype, adding to evidence for pathogenicity of this trinucleotide repeat size.
\end{abstract}

Keywords: Spinocerebellar ataxias; Trinucleotide repeat expansion; Molecular genetics; Heredodegenerative disorders; Nervous system

\section{Introduction}

Spinocerebellar ataxia type 6 (SCA6) is one of multiple autosomal dominant progressive ataxias due to unstable trinucleotide repeat gene sequences. Overall prevalence of SCA6 is estimated to be 5:100,000 [1]. Carrier rates of the full-penetrance allele vary by geographic location, presumably due to founder effect, with the highest frequency observed in Japan (about 30\%) followed by the United States and Germany; much of the literature describing affected individuals originates from Japan [2].

The principal feature of hereditary ataxias is progressive gait and limb ataxia with dysarthria and other progressive neurological deficits due to cerebellar and brainstem degeneration [3]. SCA6 is characterized by the triad of adult-onset, slowly progressive cerebellar ataxia, dysarthria, and nystagmus, with a mean age of onset of approximately 40 to 50 years [4]. Initial symptoms are imbalance and gait unsteadiness in approximately $90 \%$ of individuals; the remainder present with dysarthria. Signs and symptoms progress slowly, though eventually all affected persons have ataxia, upper limb in coordination, intention tremor, and dysarthria. Dysphagia and choking are common. Visual disturbances and eye movement disorders including horizontal or vertical gaze nystagmus and diplopia develop in the majority of individuals with SCA6, in contradistinction to fewer than $10 \%$ of those with other forms of SCA [5]. Hyperreflexia and extensor plantar responses occur in up to $50 \%$ of individuals with SCA6. Basal ganglia signs, such as dystonia and blepharospasm, are noted in a quarter of affected individuals. Cognition is generally preserved: neuropsychological testing of affected individuals has revealed no significant cognitive deficits [6]. Brain imaging is characterized by global cerebellar atrophy.

Management for SCA6 is supportive and includes regular neurologic evaluations; physical, occupational, and speech therapy; home and workplace modification; and supportive and assistive devices. Medications such as the carbonic anhydrase inhibitor acetazolamide may aid in decreasing ataxia, and vestibular suppressants (anticholinergics, antihistamines, benzodiazepines, and calcium channel antagonists) may reduce vertigo. It is advised to avoid alcohol and sedative-hypnotics that would exacerbate incoordination [4].
The only gene thus far associated with SCA6 is CACNA1A, located on chromosome 19 at 19 p13.2 encoding the transmembrane poreforming subunit of the P/Q-type or CaV2.1 voltage-gated calcium channel (VGCC). CACNA1A contains a polymorphic trinucleotide (CAG) repeat tract in exon 47 that is meiotically unstable and may expand during gametogenesis, particularly oogenesis (Figure 1).

The disease mechanism of SCA6 is postulated to be neuronal degeneration due to cytoplasmic aggregates of the calcium channel protein attributable to increased glutamine residues encoded by the expansion of the CAG codon in CACNA1A [7]. This polyglutamine mutation is a feature common to trinucleotide repeat expansionmediated neurodegenerative disorders. Abnormal calcium trafficking due to mutation in the calcium channel has also been proposed as a component of SCA6 pathogenesis [8].

Allelic disorders due to point mutation in CACNA1A include episodic ataxia type 2, familial hemiplegic migraine, and familial hemiplegic migraine with progressive cerebellar ataxia. Point mutation in CACNA1A has also been described in a case of congenital cerebellar atrophy with family history of hemiplegic migraine [9]. Normal CACNA1A alleles contain 18 or fewer CAG repeats [10]

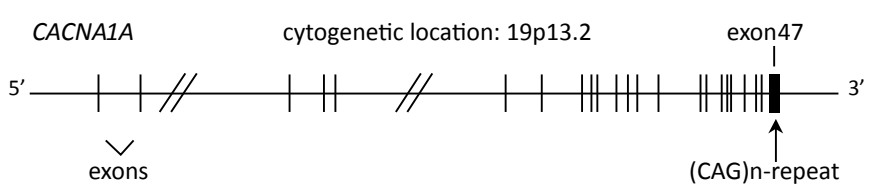

Figure 1: Schematic of CACNA1A gene.

*Corresponding author: Leslie Manace Brenman, University of California Sanfrancisco, California, USA, Tel: 011-1-510-752-2029; Fax: 011-1-510-7526754; E-mail: leslie.manace@kp.org

Received February 12, 2013; Accepted February 26, 2013; Published February 27, 2013

Citation: Brenman LM (2013) Spinocerebellar Ataxia Type 6 (SCA6) Phenotype in a Patient with an Intermediate Mutation Range CACNA1A Allele. J Neurol Neurophysiol 4: 144. doi:10.4172/2155-9562.1000144

Copyright: (C) 2013 Brenman LM. This is an open-access article distributed unde the terms of the Creative Commons Attribution License, which permits unrestricted use, distribution, and reproduction in any medium, provided the original author and source are credited. 
and full penetrance pathogenic alleles contain 20 to 33 CAG repeats $[11,12]$. Nineteen triplets in CACNA1A are currently classified as an "intermediate" or "mutable normal" finding.

\section{Case Report}

\section{Clinical presentation}

G.C. is a 44-year-old woman of Korean descent with a 10-year history of worsening tremor who was referred to medical genetics to review the clinical significance of spinocerebellar ataxia genetic testing.

The patient first noticed an asymmetric hand tremor that was worse on her dominant (right) side at 34 years of age. She was living in Germany at the time, and presented to neurology there. She reportedly underwent brain magnetic resonance imaging (MRI) with normal findings and was prescribed propranolol and primidone (unknown dosage) that-individually and in combination-initially alleviated the tremor but lost efficacy over time (records not available for review). The patient also used cannabis (unknown dosage) with partial relief of tremor symptoms. G.C. moved to the United States at 39 years, and by 40 years of age the tremor had worsened to the point that she was no longer able to work.

By her evaluation in genetics at 44 years, the patient could not write or type and had difficulty performing activities of daily living that involved implements. She had developed dysarthria, reporting that she could no longer talk as quickly or clearly as usual. She noted occasional blepharospasm, though denied nystagmus or diplopia. It was difficult for the patient to raise herself from a seated position. She described herself as increasingly clumsy, and had had multiple falls.

\section{Past pedical history}

Migraine headaches began at 40 years of age, occurred approximately monthly, and was aggravated by light; treatment included ibuprofen, Fioricet (acetominophen, butalbital and caffeine), gabapentin, topiramate, clonazepam, and acupuncture. The patient was also diagnosed with irritable bowel syndrome, allergic rhinitis, and temporomandibular joint disorder.

\section{Social history}

The patient lives with her husband and has two adult children. She has used, and continues to use, cannabis in tablet and inhaled form at intervals between every few months and every week. She denied use of other tobacco products, illicit drugs, or excess alcohol.

\section{Family history}

The patient is one of 4 siblings. Her mother, of Korean descent, died at 59 years of an unspecified pulmonary condition without known history of tremor, ataxia, or nystagmus. Her father, of Korean descent, is alive at 80 years of age without known tremor, ataxia, or nystagmus. Consanguinity was denied. The patients' siblings, in their 40 s to 50 s, are reportedly well without neurologic problems. There is a paternal aunt in her 70s with possible mild tremor; she has used acupuncture for some years to address the complaint. The age of onset in this aunt and any diagnostic work-up is unknown. The patient has a son who is 22 years of age and well, and a daughter who is 20 years old and well; neither has children. There is no known diagnosis of spinocerebellar ataxia in relatives. The family history was reported by the patient; relatives' medical records were not available for review.

\section{Physical examination}

On initial neurologic evaluation in the United States when the patient was 40 years of age, G.C. was found to be alert and oriented with a normal speech pattern. She had normal cranial nerve assessment without nystagmus noted. Normal symmetric tone and strength without atrophy, rigidity, spasticity, or fasciculations were noted. Cerebellar exercises including gait and speech observation and rapid alternating movements were unremarkable, and deep tendon reflexes were symmetric and normal. She demonstrated normal sensation and balance. There was a rapid and fine tremor of mostly the right hand that with skilled movements became coarse: the handwriting tremor was so violent that her writing was illegible.

On follow-up with neurology at 44 years of age, the patient presented with worsened hand tremor and more frequent migraine headaches. Physical examination at this time revealed a wide-spaced stance, unsteadiness on standing up from a seated position, and difficulty with tandem gait. There was bilateral hand essential and coarse intention tremor. Blood work included normal vitamin B12, thyroid function tests, and electrolytes. Fasting glucose was slightly elevated at $109 \mathrm{mg} / \mathrm{dl}$. MRI of the brain was repeated with minimal white matter signal changes in the right centrum semi-ovale and otherwise normal findings, including unremarkable appearance of the cerebellum.

Physical exam with genetics at 44 years revealed no dysmorphic features. The patient had irregularity of voice volume and word clarity. There was normal muscle bulk. Hand tremor was present bilaterally that worsened on hand use. The patient demonstrated imbalance on raising herself from a seated position and standing. She walked with a slow, ataxic gait.

\section{Genetic testing}

Ataxia gene panel testing by peripheral blood sample was completed consisting of: Aprataxin sequencing; DRPLA targeted analysis; Friedreich sequencing and targeted testing; MIRAS-specific POLG1 targeted testing; SCA1 targeted testing, SCA2 expansion analysis, SCA3 targeted testing, SCA5 sequencing, SCA6 targeted testing, SCA7 expansion analysis, SCA8, SCA10, SCA12 (PPP2R2B), SCA13 select exon testing, SCA14 targeted testing, SCA17 targeted testing, SCA28 (AFG3L2) sequencing; TTPA sequencing; and SIL1 sequencing (Athena Diagnostics Laboratory). The testing revealed a "borderline" finding of 13 and 19 CAG repeats in codon 2314 of CACNA1A (normal less than or equal to 18 repeats); all remaining gene analysis was normal. The method of analysis of CACNA1A was PCR amplification of highly purified genomic DNA and automated unidirectional DNA sequencing of entire coding region (47 exons of Genbank NM_023035 plus exons $31 \mathrm{~B}$ and $37 \mathrm{~B}$ and additional alternative published splice sites adjacent to some exons; $7518 \mathrm{bp}$ ) including the highly conserved flanking intronic sequences of the exon-intron splice junctions for all 47 exons. The abnormal sequence variant was confirmed by bidirectional sequencing. Repeat analysis of CACNA1A trinucleotide repeat region by PCR analysis of genomic DNA extracted from EDTA blood was completed, confirming the 13 and 19 CAG repeats (The Permanente Medical Group Regional Genetics Laboratory).

\section{Discussion}

SCA6 is among the more common of the trinucleotide repeat expansion-mediated hereditary ataxias. Individuals affected with SCA6 have been reported with CAG repeat expansion in either one or both CACNA1A alleles. Presence of CACNA1A triplet expansion in the homozygous state has been reported to cause a more severe phenotype and earlier onset of symptoms [13-15]. There is a direct correlation between age of onset of SCA6 and the sum of a patient's two CACNA1A 
Citation: Brenman LM (2013) Spinocerebellar Ataxia Type 6 (SCA6) Phenotype in a Patient with an Intermediate Mutation Range CACNA1A Allele. J Neurol Neurophysiol 4: 144. doi:10.4172/2155-9562.1000144

$(\mathrm{CAG})_{\mathrm{n}}$ repeat sizes [16]. SCA6 demonstrates variable expression: individuals carrying the same size full-penetrance CACNA1A alleles may display divergent age of onset and clinical course $[4,11]$.

The pathogenicity of 19 CAG repeats in CACNA1A appears to be variable. Families have been described with meiotic expansion from the 19-CAG repeat allele to pathogenic range causing the SCA6 phenotype [17]. Multiple individuals affected with SCA6 have been found to be homozygous for this intermediate 19-CAG repeat allele [18]. Individuals with atypical features of SCA6 or ataxia without other findings of SCA6 have been reported to carry the 19-CAG repeat allele in heterozygous state $[19,20]$. However, asymptomatic individuals (up to 75 years of age) heterozygous for 19 CAG repeats in the CACNA1A gene have also been reported [20]. The differing clinical correlation between 19 CAG repeats in CACNA1A and an ataxia phenotype suggest a dose-dependent and variably penetrant role of the allele.

The patient presented in this case was carries 19 CAG repeats in CACNA1A in the absence of point mutation in the remainder of the gene. Genetic testing for related neurodegenerative diseases was negative. The findings suggest a pathogenic role for the 19-CAG repeat allele in heterozygous state in this patient. Limitations of the interpretation of the CACNA1A result in this case include the possibility of other currently undetectable mutations in CACNA1A or regulatory regions; epistatic or other modifying effects; and potential locus heterogeneity. The age of onset and course of the patient's signs and symptoms is compelling for SCA6, however there is not pathologic confirmation for SCA6 available apart from clinical phenotype and supportive molecular genetic results; the patient did not yet display typical global cerebellar atrophy on MRI brain study. The lack of a positive family history or ability to test other relatives poses additional limitations.

Genetic counseling for family members, notably the patient's adult children, would entail discussion of the risk for expansion of the patient's CACNA1A trinucleotide tract to pathogenic range and the chance of carrying the same "intermediate" 19-CAG repeat CACNA1A gene. Predictive $C A C N A 1 A$ triplet repeat genetic testing should be accompanied by lengthy pre-test counseling with genetics regarding the benefits and risks of identifying susceptibility to neurodegenerative disease for which there is only supportive management available at this point. Should the patient's children elect to pursue SCA6 testing and demonstrate the same 19-CAG repeat allele, discussion regarding the unclear effect of this variant would be important, describing the possible outcomes of being affected or unaffected with some or all components of SCA6 over time. Preconception counseling regarding the risk for trinucleotide tract expansion and affected offspring would also be indicated.

As with many genetic markers, the 19-CAG repeat CACNA1A allele may represent a susceptibility factor with variable penetrance and expression depending on other modifying variables. Heterozygous carrier state for 19 CAG repeats in CACNA1A continues to be classified as "intermediate," similar to "gray zone" or "borderline" tracts in other trinucleotide repeat expansion diseases such as fragile $\mathrm{X}$ syndrome. Additional reports of carriers with 19 CAG repeats in CACNA1A and their corresponding phenotypes will likely be increasingly available as molecular genetics panels and whole exome/genome analysis become prevalent, and will add to our understanding of the molecular basis for this spinocerebellar ataxia subtype.

\section{Acknowledgements}

The author thanks Dr. Christopher M. Gomez, Brent L. Fogel, Dr. Xiao-Dong
Han, and Dr. Sat Dev Batish for molecular genetics and medical information.

\section{References}

1. Craig K, Keers SM, Archibald K, Curtis A, Chinnery PF (2004) Molecular epidemiology of spinocerebellar ataxia type 6. Ann Neurol 55: 752-755.

2. Matsumura $R$, Futamura $N$, Fujimoto $Y$, Yanagimoto $S$, Horikawa $H$, et al (1997) Spinocerebellar ataxia type 6. Molecular and clinical features of 35 Japanese patients including one homozygous for the CAG repeat expansion. Neurology 49: 1238-1243.

3. Schmitz-Hübsch T, Coudert M, Bauer P, Giunti P, Globas C, et al. (2008) Spinocerebellar ataxia types $1,2,3$, and 6 : disease severity and nonataxia symptoms. Neurology 71: 982-989.

4. Gomez CM, Thompson RM, Gammack JT, Perlman SL, Dobyns WB, et al. (1997) Spinocerebellar ataxia type 6: gaze-evoked and vertical nystagmus Purkinje cell degeneration, and variable age of onset. Ann Neurol 42: 933-950.

5. Yabe I, Sasaki H, Matsuura T, Takada A, Wakisaka A, et al. (1998) SCA6 mutation analysis in a large cohort of the Japanese patients with late-onse pure cerebellar ataxia. J Neurol Sci 156: 89-95.

6. Globas C, Bösch S, Zühlke Ch, Daum I, Dichgans J, et al. (2003) The cerebellum and cognition. Intellectual function in spinocerebellar ataxia type 6 (SCA6). J Neurol 250: 1482-1487.

7. Ishikawa K, Fujigasaki H, Saegusa H, Ohwada K, Fujita T, et al. (1999) Abundant expression and cytoplasmic aggregations of [alpha]1 $\mathrm{A}$ voltagedependent calcium channel protein associated with neurodegeneration in spinocerebellar ataxia type 6. Hum Mol Genet. 8: 1185.

8. Restituito S, Thompson RM, Eliet J, Raike RS, Riedl M, et al. (2000) The polyglutamine expansion in spinocerebellar ataxia type 6 causes a beta subunit-specific enhanced activation of P/Q-type calcium channels in Xenopus oocytes. J Neurosci 20: 6394-6403.

9. Naik S, Pohl K, Malik M, Siddiqui A, Josifova D (2011) Early-onset cerebellar atrophy associated with mutation in the CACNA1A gene. Pediatr Neurol 45 328-330.

10. Shizuka M, Watanabe M, Ikeda Y, Mizushima K, Okamoto K, et al. (1998) Molecular analysis of a de novo mutation for spinocerebellar ataxia type 6 and (CAG)n repeat units in normal elder controls. J Neurol Sci 161: 85-87.

11. Jodice C, Mantuano E, Veneziano L, Trettel F, Sabbadini G, et al. (1997) Episodic ataxia type 2 (EA2) and spinocerebellar ataxia type 6 (SCA6) due to CAG repeat expansion in the CACNA1A gene on chromosome 19p. Hum Mol Genet 6: 1973-1978.

12. Yabe I, Sasaki H, Takeichi N, Takei A, Hamada T, et al. (2003) Positiona vertigo and macroscopic downbeat positioning nystagmus in spinocerebellar ataxia type 6 (SCA6). J Neurol 250: 440-443.

13. Matsuyama Z, Kawakami H, Maruyama H, Izumi Y, Komure O, et al. (1997) Molecular features of the CAG repeats of spinocerebellar ataxia 6 (SCA6) Hum Mol Genet 6: 1283-1287.

14. Ikeuchi T, Takano H, Koide R, Horikawa Y, Honma Y, et al. (1997) Spinocerebellar ataxia type 6: CAG repeat expansion in alpha1A voltagedependent calcium channel gene and clinical variations in Japanese population Ann Neurol 42: 879-884.

15. Geschwind DH, Perlman S, Figueroa KP, Karrim J, Baloh RW, et al. (1997) Spinocerebellar ataxia type 6 . Frequency of the mutation and genotypephenotype correlations. Neurology 49: 1247-1251.

16. Takahashi H, Ishikawa K, Tsutsumi T, Fujigasaki H, Kawata A, et al. (2004) A clinical and genetic study in a large cohort of patients with spinocerebellar ataxia type 6. J Hum Genet 49: 256-264.

17. Shimazaki H, Takiyama Y, Sakoe K, Amaike M, Nagaki H, et al. (2001) Meiotic instability of the CAG repeats in the SCA6/CACNA1A gene in two Japanese SCA6 families. J Neurol Sci 185: 101-107.

18. Mariotti C, Gellera C, Grisoli M, Mineri R, Castucci A, et al. (2001) Pathogenic effect of an intermediate-size SCA-6 allele (CAG)(19) in a homozygous patient. Neurology 57: 1502-1504.

19. Katayama T, Ogura Y, Aizawa H, Kuroda H, Suzuki Y, et al. (2000) Nineteen CAG repeats of the SCA6 gene in a Japanese patient presenting with ataxia J Neurol 247: 711-712. 
Citation: Brenman LM (2013) Spinocerebellar Ataxia Type 6 (SCA6) Phenotype in a Patient with an Intermediate Mutation Range CACNA1A Allele. J Neurol Neurophysiol 4: 144. doi:10.4172/2155-9562.1000144

Page 4 of 4

20. Ishikawa K, Tanaka H, Saito M, Ohkoshi N, Fujita T, et al. (1997) Japanese families with autosomal dominant pure cerebellar ataxia map to chromosome 19p13.1-p13.2 and are strongly associated with mild CAG expansions in the spinocerebellar ataxia type 6 gene in chromosome 19p13.1. Am J Hum Genet 61: $336-346$ 